\title{
Ciclo de mejora en el estudio de la gestionabilidad de la tecnología termosolar cilindroparabólica en el mercado energético
}

\section{Cycle of improvement in the study of the dispatchability of the parabolic trough technology in the energy market}

CRISTINA PRIETO Ríos

ORCID: https://orcid.org/0000-0002-9300-6967

Universidad de Sevilla

Departamento de Ingeniería Energética

cprieto@us.es

Fecha de recepción:

Fecha de aceptación:

DOI: http://dx.doi.org/10.12795/9788447221912.111

Pp.: 2474-2492 


\section{Resumen:}

El trabajo muestra el ciclo de mejora implementado en la asignatura de Centrales Solares del Grado de Ingeniería de la Energía, y en concreto, en la tecnología de concentración cilindropabólica. El objetivo del ciclo era que los alumnos llegaran a comprender dos conceptos fundamentales de la tecnología; el primero era que la energía termosolar es una solución energética renovable y gestionable que da respuesta a las necesidades del mercado energético. En segundo lugar, que los alumnos comprendieran que los sistemas de almacenamiento térmico son el componente clave de la tecnología que permite ajustar la curva de producción a la curva de demanda, incluso cuando no hay recurso solar disponible. Tras la implementación de un modelo metodológico basado en las ideas de los alumnos y de las alumnas y en las actividades de contrate, el ciclo de mejora implementado ha cumplido con los objetivos establecidos.

Palabras claves: Centrales Solares, Ingeniería de la Energía, Docencia Universitaria, Experimentación Docente Universitaria, Almacenamiento de Energía

\section{Summary}

The work shows the cycle of improvement implemented in the subject of Solar Power Plants of the Degree of Energy Engineering, and specifically, in the technology of parabolic trough collector. The objective of the cycle was to understand two fundamental concepts of this technology; the first was that solar thermal energy is a renewable and dispatchable energy solution that responds to the needs of the energy market. Second, the students must understand that thermal storage systems are the key component of the technology that allows to adjust the curve of production and the demand of demand, even when there is no solar resource available. After the implementation of a methodological model based on the ideas of the students and the contracting activities, the improvement cycle implemented has fulfilled the established objectives.

Keywords: Solar Plants, Energy Engineering, University Teaching, Energy Storage

Jornadas de Formación e Innovación Docente del Profesorado | № 2 (2019) Esta obra se distribuye con la licencia Creative Commons 


\section{Contexto}

Se aplica el ciclo mejora en la asignatura de Centrales Solares, del 4으 curso del Grado de la Energía. La clase la forman 45 alumnos, cuya asistencia se ha controlado ya que, aunque no era obligatoria, se puntuaba junto a la participación. En el ciclo de mejora en el Aula (CIMA) se ha buscado en todo momento que los alumnos aprendieran a pensar en la resolución de problemas desde un aspecto técnico aplicado, tal y como tendrán que hacerlo en la vida laboral a la que van a acceder en breve. El desarrollo del ciclo se ha llevado a cabo en un ambiente participativo y proactivo por parte de los asistentes, con una duración de 8 horas.

El objetivo conceptual del tema elegido es llegar a comprender dos conceptos fundamentales de la tecnología cilindroparabólica:

- La energía termosolar es una solución energética renovable y gestionable que da respuesta a las necesidades del mercado energético

- Los sistemas de almacenamiento térmico son el componente clave de la tecnología que permite ajustar la curva de producción a la curva de demanda, incluso cuando no hay recurso solar disponible.

\section{Diseño previo del CIMA}

\section{Mapa de contenido y problemas}

En la figura 1 se observa el mapa de contenido y problemas de la parte de la asignatura donde se implementa el CIMA, en él se enlazan los contenidos conceptuales, procedimentales y actitudinales objetivos del tema. A través de este mapa se busca dar respuesta a estos preguntas-problemas claves: 
1. ¿Cómo puedo aumentar la producción eléctrica en una planta existente?

2. Si el precio de la energía nocturna es dos veces mayor ¿Cómo puedo aumentar las ganancias?

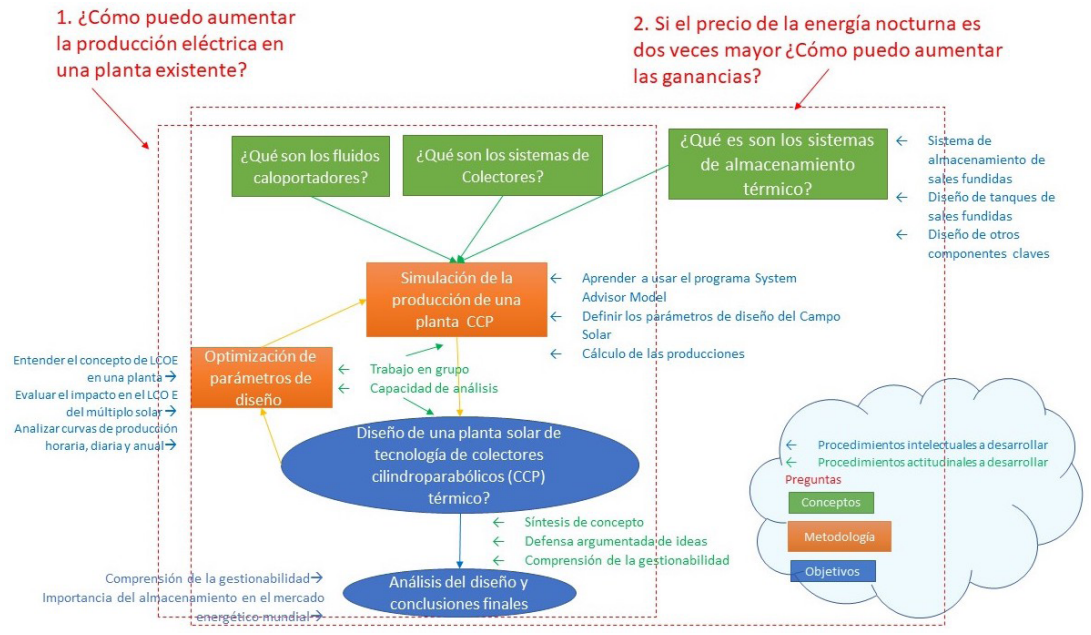

Figura 1 Mapa de contenidos y problemas del CIMA

Estas preguntas permiten realizar la evaluación del CIMA a través de la escalera que comentaremos en un apartado posterior.

\section{Modelo metodológico posible}

Este CIMA se aplica en dos clases de 4 horas cada una. En cada clase se sigue un modelo en el que los alumnos trabajara en grupos para dar respuesta a las preguntas guía y donde serán los protagonistas (Finkel, 2008).

En una primera sesión de 4 horas, se parte de una fase Teórica. A continuación se pasa a una fase Práctica lanzando una pregunta que será el hilo conductor: ¿Cómo puedo aumentar la producción eléctrica en una planta existente? Los alumnos trabajaran en grupos analizando la planta existente y como mejorarla. Se establece una fase de generación de Ideas de los Alumnos y de las Alumnas y 
se continúa con una Actividad de Contraste. Una vez finalizada esta primera etapa de debate (con planta modelada y analizada), se reinicia el proceso. Se lanza la segunda pregunta conductora: Si el precio de la energía nocturna es dos veces mayor ¿Cómo puedo aumentar las ganancias? Los alumnos iniciaran una fase Práctica de trabajo fuera del aula para darle respuesta.

En la segunda sesión de 4 horas, se continúa con la fase Práctica con una Actividad de Contraste donde se comparan y se discuten los trabajos realizados por los alumnos. Se termina la sesión con una fase Teórica donde se explica el concepto desarrollado en la sesión, la gestionabilidad a través del almacenamiento térmico, concepto que ha servido para dar respuesta a las preguntas planteadas. Finalmente, se lleva a cabo una fase de Conclusiones de las dos sesiones.
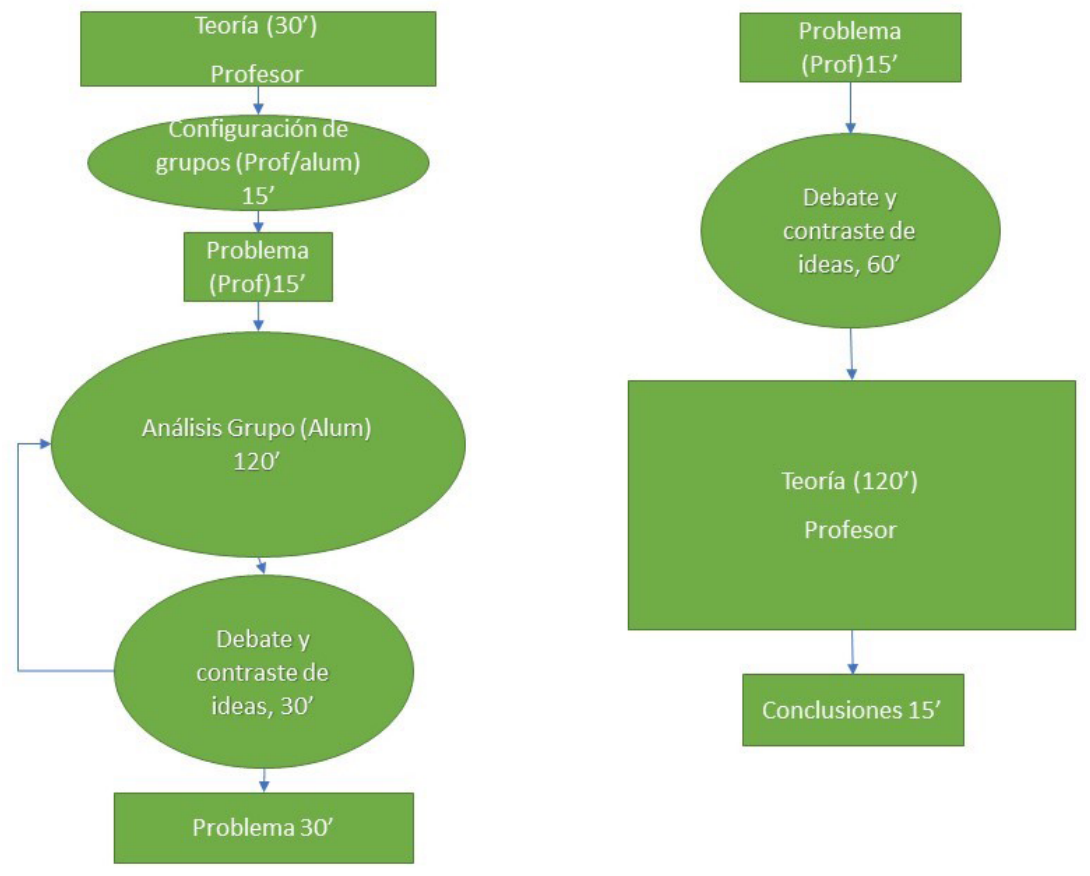

Figura 2: Esquema de la metodología (8h)

Jornadas de Formación e Innovación Docente del Profesorado | № 2 (2019) Esta obra se distribuye con la licencia Creative Commons Reconocimiento-NoComercial-SinObraDerivada 4.0 Internacional (CC BY-NC-ND 4.0.) 


\section{Secuencia de actividades:}

En las tablas 1 y 2 se recoge la secuencia de actividades propuestas para las dos sesiones del CIMA que buscan facilitar el proceso de aprendizaje (Porlán, 2017).

Tabla 1: Secuencia de actividades de la primera sesión del CIMA

\begin{tabular}{|c|c|c|}
\hline ACTIVIDADES & ALCANCE & Tiempo \\
\hline Cuestionario & Se entrega cuestionario & $10^{\prime}$ \\
\hline \multicolumn{3}{|c|}{ Recursos: Se entregará cuestionario en un folio. Se pedirá con nombre } \\
\hline \multicolumn{3}{|l|}{ Teoría $\left(30^{\prime}\right)$} \\
\hline Actividad 1 & $\begin{array}{l}\text { Se hace un resumen de los componentes } \\
\text { de la tecnología vistos hasta la fecha del } \\
\text { CIMA }\end{array}$ & $30^{\prime}$ \\
\hline \multicolumn{3}{|c|}{$\begin{array}{l}\text { Recursos: presentación } \\
\text { en power point } \\
\text { proyectada }\end{array}$} \\
\hline \multicolumn{3}{|l|}{ Problema $\left(30^{\prime}\right)$} \\
\hline Actividad 2 & $\begin{array}{l}\text { Se explica que se va a trabajar en grupo } \\
\text { para hacer un análisis del diseño de las } \\
\text { plantas. Se constituirán grupos de } 2 \text { a } 4 \\
\text { personas. }\end{array}$ & $15^{\prime}$ \\
\hline \multicolumn{3}{|c|}{ Los grupos trabajaran con ordenador } \\
\hline Actividad 3 & $\begin{array}{l}\text { Se lanzará pegunta ¿Cómo puedo aumentar } \\
\text { la producción eléctrica en una planta } \\
\text { existente? }\end{array}$ & \\
\hline Actividad 4 & $\begin{array}{l}\text { Se hará entrega de los datos de una planta } \\
\text { existente y se les indicará el software a } \\
\text { utilizar para el proceso de optimización. }\end{array}$ & $15^{\prime}$ \\
\hline \multicolumn{3}{|c|}{$\begin{array}{r}\text { Recursos: Se deberán instalar el programa SAM para modelar plantas, ficha con } \\
\text { datos de planta existente y se entregará tutorial del programa }\end{array}$} \\
\hline Ideas de los a & jo en $\operatorname{Grupo}\left(60^{\prime}\right)$ & \\
\hline
\end{tabular}

Jornadas de Formación e Innovación Docente del Profesorado | № 2 (2019) Esta obra se distribuye con la licencia Creative Commons Reconocimiento-NoComercial-SinObraDerivada Internacional (CC BY-NC-ND 4.0.) 


\begin{tabular}{|c|c|c|}
\hline Actividad 5 & $\begin{array}{l}\text { Los alumnos deberán modelar la planta } \\
\text { de referencia. Deberán dar respuesta a los } \\
\text { puntos: } \\
\text { · } \text { Factor de capacidad } \\
\text { - Producción anual } \\
\text { - } \quad \text { LCOE } \\
\text { La profesora irá trabajando con los grupos } \\
\text { para resolver los problemas asociado al } \\
\text { modelado de las plantas en el simulador. } \\
\text { Las dudas repetidas se explicarán en voz } \\
\text { alta para su explicación conjunta. }\end{array}$ & $60^{\prime}$ \\
\hline \multicolumn{3}{|c|}{ Recursos: Se usará el programa SAM instalado } \\
\hline \multicolumn{3}{|c|}{ Contraste de ideas $\left(90^{\prime}\right)$} \\
\hline Actividad 6 & $\begin{array}{l}\text { Se comparar los resultados obtenidos en } \\
\text { la actividad anterior. Se pondrán en común } \\
\text { modelos simulados de la planta existente }\end{array}$ & $15^{\prime}$ \\
\hline \multicolumn{3}{|c|}{ Recursos: Ordenadores por grupo y pizarra } \\
\hline Actividad 7 & $\begin{array}{l}\text { Tras validar la simulación de la planta } \\
\text { de referencia, los alumnos deben dar } \\
\text { respuesta a la pregunta realizada. } \\
\text { Los alumnos deberán analizar en grupo } \\
\text { que parámetros se modifican para mejorar } \\
\text { la planta. En cada modificación deberán } \\
\text { analizar: } \\
\text { - Factor de capacidad } \\
\text { - Producción anual } \\
\text { - } \quad \text { LCOE }\end{array}$ & $60^{\prime}$ \\
\hline \multicolumn{3}{|c|}{$\begin{array}{r}\text { Recursos: Se entrega ficha de la planta a modelar y ficha de puntos a analizar: Factor } \\
\text { de capacidad, producción anual, LCOE }\end{array}$} \\
\hline Actividad 8 & $\begin{array}{l}\text { Escribir en la pizarra los resultados } \\
\text { analizados, discutiendo entre todos los } \\
\text { efectos de los cambios propuestos }\end{array}$ & $15^{\prime}$ \\
\hline \multicolumn{3}{|c|}{ Recursos: Pizarra } \\
\hline \multicolumn{3}{|c|}{ Problema $\left(30^{\prime}\right)$} \\
\hline Actividad 9 & $\begin{array}{l}\text { Una vez se haya analizado como se } \\
\text { modifica una planta para mejorar su } \\
\text { producción, se lanza la segunda pregunta: } \\
\text { Si el precio de la energía nocturna es dos } \\
\text { veces mayor ¿Cómo puedo aumentar las } \\
\text { ganancias? } \\
\text { Se establece las bases para llevar a cabo } \\
\text { este trabajo en casa }\end{array}$ & $30^{\prime}$ \\
\hline
\end{tabular}

Jornadas de Formación e Innovación Docente del Profesorado I № 2 (2019) Esta obra se distribuye con la licencia Creative Commons Reconocimiento-NoComercial-SinObraDerivada Internacional (CC BY-NC-ND 4.0.) 
Tabla 2 Secuencia de actividades de la segunda sesión del CIMA

\begin{tabular}{|c|c|c|}
\hline \multicolumn{3}{|c|}{ Problema $\left(15^{\prime}\right)^{\prime}$} \\
\hline Actividad 10 & $\begin{array}{l}\text { Se hace recordatorio de la clase anterior y se recuerda } \\
\text { la pregunta que se lanzó: Si el precio de la energía } \\
\text { nocturna es dos veces mayor ¿Cómo puedo aumentar } \\
\text { las ganancias? }\end{array}$ & $15^{\prime}$ \\
\hline \multicolumn{3}{|c|}{$\begin{array}{l}\text { Recursos: Se pide que respondan a la ficha de puntos a analizar: datos de } \\
\text { producción anual, distribución de costes, cálculo del múltiplo Solar, propiedades de } \\
\text { colector, CAPEX y LCOE }\end{array}$} \\
\hline \multicolumn{3}{|c|}{ Contrate $\left(60^{\prime}\right)$} \\
\hline Actividad 11 & $\begin{array}{l}\text { Se comparan en la pizarra los resultados obtenidos por } \\
\text { los grupos. Cada grupo expondrá sus resultados }\end{array}$ & $60^{\prime}$ \\
\hline \multicolumn{3}{|c|}{ Recursos: Pizarra } \\
\hline \multicolumn{3}{|l|}{ Teoría $\left(120^{\prime}\right)$} \\
\hline Actividad 12 & $\begin{array}{l}\text { La profesora explicará el concepto de gestionabilidad. } \\
\text { Se pondrán las bases de los conceptos que se han } \\
\text { esbozado en el trabajo en grupo. } \\
\text { Explicará las necesidades del mercado para ajustar } \\
\text { curva de consumo y la de demanda } \\
\text { Explicar los sistemas de almacenamiento térmico y sus } \\
\text { componentes }\end{array}$ & $120^{\prime}$ \\
\hline \multicolumn{3}{|c|}{$\begin{array}{r}\text { Recursos: PPT, ordenadores por grupo y videos de plantas con almacenamiento en } \\
\text { sales fundidas. }\end{array}$} \\
\hline \multicolumn{3}{|c|}{ Resumen y conclusiones (30') } \\
\hline Actividad 13 & $\begin{array}{l}\text { Resumir los datos anteriores y establecer las } \\
\text { conclusiones. }\end{array}$ & $20^{\prime}$ \\
\hline Actividad 14 & Cuestionario final & $10^{\circ}$ \\
\hline
\end{tabular}

\section{Cuestionario}

Tal como se indica en la secuencia de actividades, se les entrega un cuestionario inicial con el objetivo de evaluar el punto de partida de cada alumno y de cada alumna respecto a los contenidos a impartir. Se busca que vean la 
situación práctica a la que da respuesta el contenido a impartir. A continuación, se incluye el texto del cuestionario:

"Somos una empresa de consultoría energética. Nos han llamado desde la empresa Abengoa e indican que necesitan nuestra ayuda. La empresa tiene tres plantas de 50 MW instaladas en Sevilla, estas plantas son de tecnología de cilindroparábolica. Las preguntas que nos plantean son:

- La empresa quiere poner una nueva planta, con la misma configuración que las existentes y nos pregunta ¿cómo pueden optimizar el diseño de esta nueva planta para minimizar los costes de producción?

- además, creen que el Gobierno va a lanzar un nuevo plan donde se va a pagar con prima extraordinaria el kWh que se genere en la franja horaria entre las 8 y las 10 de la noche. La empresa quiere saber ¿qué haría falta hacer en las plantas actuales para maximizar los beneficios?"

\section{Aplicación del CIMA}

\section{Relato de la primera sesión (4h)}

Durante la aplicación del CIMA se ha llevado un diario de clase. Las actividades se desarrollaron de manera normal, con algunos inconvenientes indicados, pero sobre todo hubo una mala previsión del tiempo, la continua participación de los alumnos como consecuencia de la interpretación cada vez mejor del concepto, hizo que se extendiera la Actividad de Contraste.

Se empieza la sesión entregando el cuestionario y se les pide que lo rellenen indicando su nombre y apellidos. Esto obliga a aclarar que no es ningún examen, sino una 
evaluación de la metodología docente, para comprender si los conceptos que se van a discutir en las horas posteriores son asimilados. Esta actividad se desarrolla sin más incidencias. Se produce cada vez más participación de los alumnos y alumnas con preguntas sobre el temario visto.

A continuación, se proyecta un resumen de los componentes de la tecnología que se han estado viendo en los días anteriores. Y en esta actividad, que duraba unos 30 minutos, se lanzó a pregunta ¿Cómo puedo aumentar la producción eléctrica en una planta existente? En este caso anticipé la pregunta a la formación de grupos, porque en la actividad 1 ya se discutió el concepto de planta como un producto demandado por el mercado para dar una solución energética.

Tal como se les comunica por enseñanza virtual (EV) el día previo, los alumnos y las alumnas han traído ordenador y tienen una idea de los grupos que quieren formar. Esto agiliza la actividad. Los asistentes se agrupan de forma libre, según ellos han decidido, y es este el momento donde surge el primer inconveniente, el aula no facilita la dinámica de trabajo en grupo. La actividad se desarrolla en un aula de filas corridas que dificulta las discusiones entre los miembros de los grupos. En cualquier caso, y tomando nota del tema logístico para futuras sesiones, se distribuyen los grupos por el aula.

A continuación, se les entrega una hoja con los datos de una planta existente y se lanza la pregunta. ¿Cómo puedo aumentar la producción eléctrica en una planta existente?. Para ello, se les explica que hay un programa de simulación de plantas que deben usar para analizar los efectos de las mejoras que propongan.

Siguiendo aproximadamente el horario estimado, a la hora de iniciarse la sesión se pide empezar a trabajar en el problema. Aquí surge el segundo inconveniente del CIMA. 
La instalación del software SAM. Aunque se les había dicho por EV que también trajeran el software instalado (es software libre), muchos de los alumnos y alumnas no lo habian hecho, lo que supuso un retraso en el inicio del análisis problema. Con unos 15 minutos de retraso se inicia el análisis, para el cual se les da una pauta de preguntas a responder.

La profesora en todo momento va de grupo en grupo resolviendo las dudas, si la duda es reiterada se responde en voz alta ya que en un primer momento son dudas de interpretación del software. A medida que los alumnos y alumnas van poniendo los datos en el simulador, empiezan a entender mejor la parte de la información que se les había dado de los componentes, ejemplo de ellos fueron las dudas sobre conceptos ya analizados en clases previas como: año meteorológico, longitudes de colector, aperturas de los mismos, separación de lazos, parámetros ópticos, refrigeración del ciclo de potencia, etc.

A la media hora de trabajar con el simulador, empiezan a calcular las primeras producciones eléctricas de la planta y empiezan a hacer sensibilidades. Empieza a mejorar el concepto de planta como producto. Una hora después, se inicia la fase de contrate. Se empieza a comparar en la pizarra los datos que han obtenido. Se comparan los datos de:

- Factor de capacidad. Esto permite mejorar el concepto de campo solar y producción. Cómo el aumento de campo es necesario para aumentar la producción eléctrica en esta planta. Se discute cómo para una parcela existente, donde no hay disponibilidad de terreno, no se podría aumentar producción porque no se puede aumentar el campo.

- Producción anual. Esta discusión permitió analizar las diferencias en función de los autoconsumos que se habían considerado. Cómo la refrigeración que considere afecta al rendimiento de ciclo y por 
tanto a la producción. También se vio el impacto de las mejoras que habían propuesto: la temperatura de salida del campo, campo de colectores con distinta concentración, etc.

- Coste nivelado de la energía. Parámetro esencial asociado a los costes de viabilidad de la tecnología.

Llegados a este punto, tuvimos que parar la discusión porque ya no nos quedaba tiempo apenas y se manda para casa que trabajen la pregunta dos. Si el precio de la energía nocturna es dos veces mayor ¿Cómo puedo aumentar las ganancias? Hubiéramos necesitado más tiempo de discusión, la comprensión del producto hizo que empezaran a plantearse dudas de los conceptos dados.

\section{Diario de la segunda sesión del CIMA (4h).}

Se inicia la segunda sesión del CIMA a la siguiente semana. Hay grupos que han venido a tutoría para discutir cómo se desplaza la producción eléctrica y dar respuesta a la pregunta lanzada, la gestionabilidad de la termosolar, y cómo es posible almacenar la energía para producir energía eléctrica en picos de demanda sin necesidad de tener recurso solar. Este es el factor clave a discutir.

La sesión empieza preguntando a los grupos como les ha ido. Hay una discusión informal sobre cómo han trabajado (15', no prevista en las actividades). Se hace recordatorio de la clase anterior y se recuerda la pregunta que se lanzó: Si el precio de la energía nocturna es dos veces mayor ¿Cómo puedo aumentar las ganancias?

De nuevo se pone en la pizarra la respuesta de los grupos y se inicia la discusión. Todos han añadido sistemas de almacenamiento para desplazar la producción, pero hay dos tendencias claras. Los que proponen añadir el almacenamiento ampliando campo solar y los que no. Este 
punto es primordial para entender los roles del almacenamiento en la termosolar. Se inicia la discusión de los efectos de modificar la producción, así como el efecto de ampliar la producción. Este último punto se interconectó con la modificación del tamaño del campo solar. Aunque llegados a este punto, el objetivo de la actividad de contraste se había acabado, los alumnos quieren modelar distintas estrategias de producción y cómo son las curvas de demanda de los distintos mercados.

Este momento marca "un antes y un después" en mi valoración de la metodología docente. A pesar de seguir pensando que clases magistrales con personal experto en la materia y buen orador es un factor importante en la docencia, en este momento me percato de la insuficiencia del método. Hasta este momento no había percibido la ausencia de la participación de los alumnos y de las alumnas en su formación. Sin embargo, el trabajo en grupo guiado por Preguntas-Clave como guía y las actividades de contraste me permite valorar la transmisión del conocimiento y recibir una información inmediata de como se está desarrollando la transferencia.

Llegados a este punto, ya llevamos más de 2 horas de clase, y voy fuera de horario para dar la parte de teoría. Propongo, por tanto, que el trabajo a entregar de la asignatura sea esta nueva iteración. Por tanto, partiendo de los valores que se dan en una oferta comercial, una potencia dada, una ubicación, con un día de diseño y una curva de demanda que les entregaré en la clase posterior, deberán diseñar una planta cilindroparabólica. Por mi parte me comprometo a realizarles una presentación del mercado energético que no estaba prevista en el temario.

Con casi $3 / 4$ de hora de retraso, inicio la presentación del último componente que nos quedaba por dar. Se les 
explica los sistemas de almacenamiento. Su concepto, clasificación y se inicia la descripción de los equipos que lo forman. En la siguiente hora y media, voy más lento de lo previsto porque hay continuas preguntas por parte de los alumnos. Además, noto que son reacios a atender a la parte más teórica y conceptual y que las preguntas se centran continuamente en la aplicación del componente.

A quince minutos de la finalización de la sesión, paro la actividad (aun me queda la mitad de la teoría) y vuelvo a pasar el cuestionario del CIMA.

Llegados a este punto he terminado el CIMA, en el cual las últimas actividades no se pudieron desarrollar, lo que me ha obligado a extenderlo a una tercera sesión de 3 horas. En esta última sesión se ha podido terminar la descripción de los componentes de los sistemas de almacenamiento, se ha discutido el mercado energético y se ha proyectado el video que se puso al inicio del cuatrimestre sobre el diseño de plantas termosolares. En esta proyección los alumnos han podido identificar por sí solos los componentes de la tecnología y su utilidad.

\section{Evaluación por escaleras}

Se ha llevado a cabo la valoración del cuestionario que se circula al inicio del CIMA y a las 8 horas de desarrollo. Aunque participan en clase 40 personas, se ha tomado solo 30 de ellas para el análisis, descartando aquellos que dejaron preguntas sin responder. La evaluación se realiza con el análisis de las escaleras de aprendizaje (Porlán, 2017). En la Figura 3 se analiza la escalera de la primera pregunta: ¿Cómo puedo aumentar la producción eléctrica en una planta existente? 


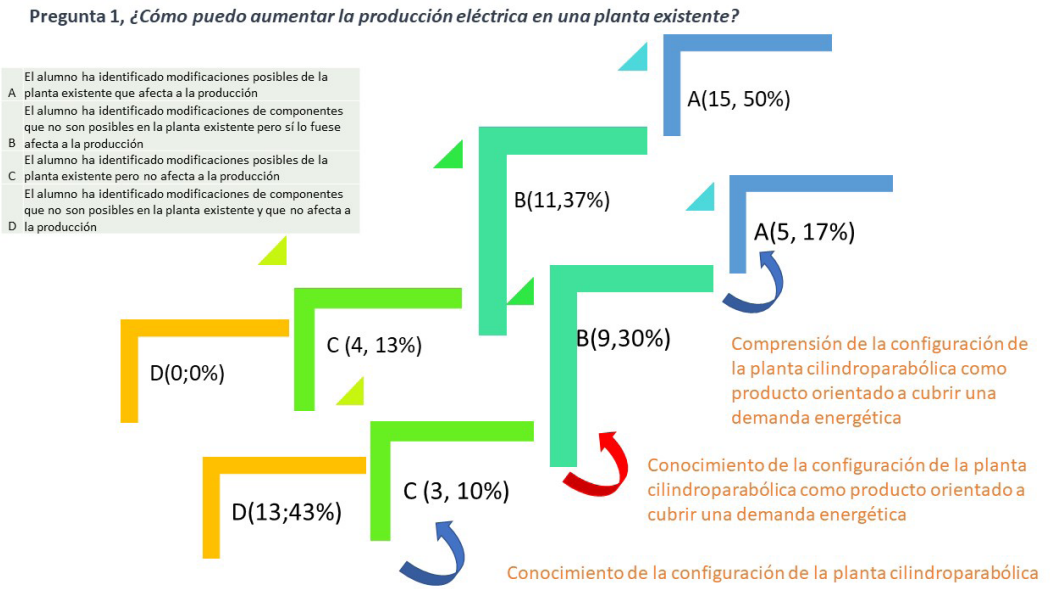

Figura 3 Escalera de aprendizaje de la pregunta 1

En el cuestionario inicial hay un $47 \%$ de alumnos que comprenden el concepto de planta como el de un producto que busca cubrir una demanda energética (respuestas $A+B)$, frente al 53\% que no lo tiene adquirido $(C+D)$. Tras el CIMA, el $87 \%$ de los alumnos y alumnas has adquirido ese conocimiento mínimo que se marcaba como objetivo de la docencia. Se adjunta en la Figura 4 como es la mejora individual, mejorando en el $\mathbf{9 7 \%}$ de los casos el conocimiento del alumno o alumna.

Pregunta 1, ¿Cómo puedo aumentar la producción eléctrica en una planta existente?

Evolución de los alumnos en la pregunta 1

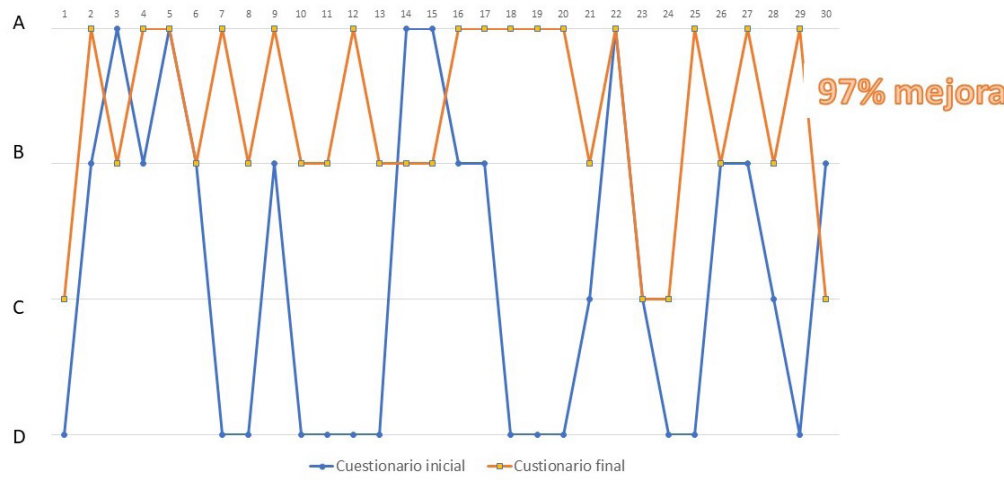

Figura 4 Evolución individual del conocimiento a la respuesta 1

Jornadas de Formación e Innovación Docente del Profesorado | № 2 (2019) Esta obra se distribuye con la licencia Creative Commons Reconocimiento-NoComercial-SinObraDerivada 4.0 Internacional (CC BY-NC-ND 4.0.) 
Si analizamos la pregunta 2, en la Figura 5 se ve la escalera de aprendizaje, donde el $82 \%$ sabía que era la gestionabilidad, aunque solo un $12 \%$ lo relacionaba con el campo solar. Tras el ciclo, el 100\% de los alumnos sabe que es la gestionabilidad, objetivo de la docencia, y el $40 \%$ lo asocia de forma explícita al campo.

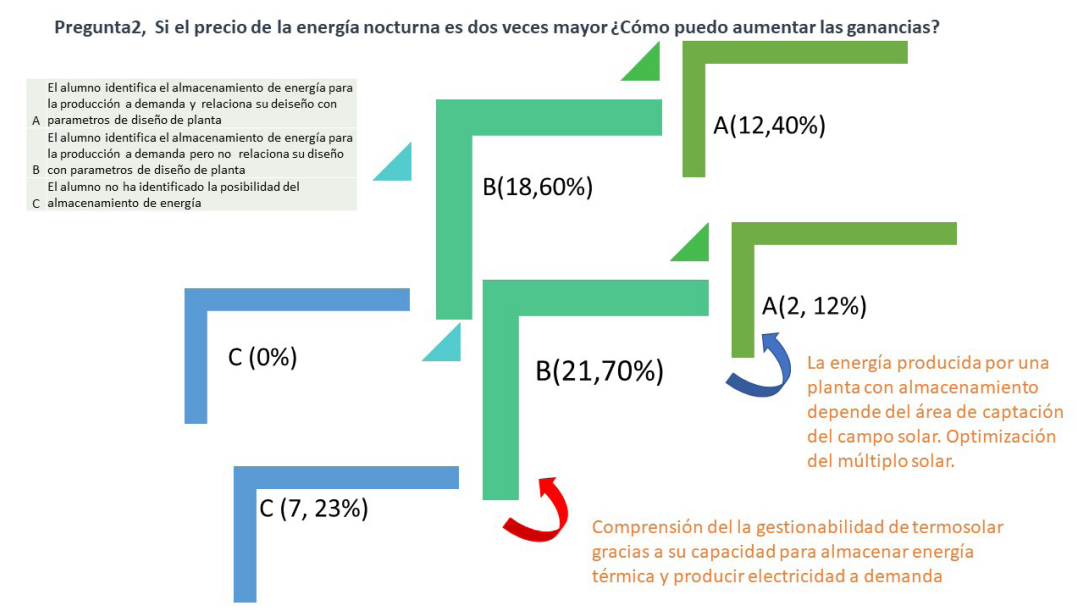

Figura 5 Escalera de aprendizaje de la pregunta 2

Se adjunta también evolución individual, con un $93 \%$ de mejora.

Pregunta2, Si el precio de la energía nocturna es dos veces mayor ¿Cómo puedo aumentar las ganancias? Evolución de los alumnos en la pregunta 2

$93 \%$ mejom

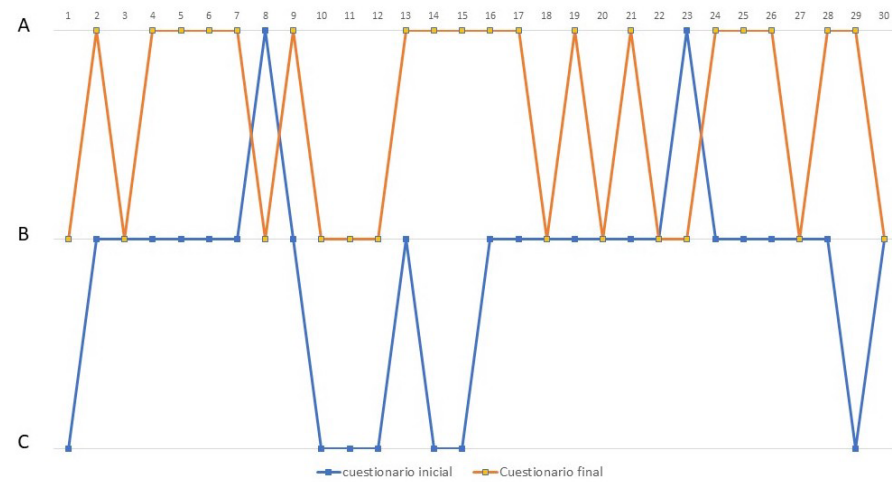

Figura 6 Evolución individual del conocimiento a la respuesta 2

Jornadas de Formación e Innovación Docente del Profesorado | № 2 (2019) Esta obra se distribuye con la licencia Creative Commons Reconocimiento-NoComercial-SinObraDerivada 4.0 Internacional (CC BY-NC-ND 4.0.) 


\section{Evaluación del CIMA}

\section{Que ha funcionado y por qué}

Claramente para mí, el modelo metodológico ha sido un éxito. La combinación de actividades de Prácticas, con Generación de Ideas de los Alumnos y Actividades de Contraste ha conseguido que se cumpla con los objetivos del mapa de contenido.

También la elaboración de dicho mapa permite ordenar y priorizar los objetivos docentes del temario a impartir ya que se ha detectado aspectos que de manera subjetiva se daban por aprendidos con la docencia previa impartida pero que no estaban incluidos en la secuencia de actividades y no estaban impartidos ni evaluados. Hay también un factor de satisfacción intrínseca en la metodología del CIMA, ya que recibes las señales de la comprensión del docente con inmediatez. Todo lo indicado invita claramente a plantearse la incorporación de la experiencia a la práctica docente habitual.

\section{Todo lo que se puede mejorar para el próxima CIMA y no debo olvidar}

En el desarrollo del CIMA también se detectan aspectos que se pueden y deben mejorar:

- La duración del CIMA estaba infraestimada gracias a la participación de los alumnos.

- La teoría impartida se debe rehacer ya que la empleada hizo que se repitieran conceptos que habíamos discutido ampliamente en la fase de análisis y contraste. Hay que reenfocar la parte teórica asumiendo que ya se ha transmitido una importante 
parte del conocimiento en la parte Práctica del método.

- Hay que estimar bien la secuencia de actividades. Un ejemplo claro es ser demasiado ambiciosa en el desarrollo de la actividad de análisis primera donde mezclé conceptos de planta con conocimientos de un software previamente no explicado. De esta forma se corre el riesgo de desviar la atención de los grupos de trabajo.

- Introducir más actividades de contraste individual, durante el CIMA.

- Reconfigurar el aula para la mejora de las actividades de contrates. 


\section{Bibliografía}

Finkel, D. (2008). Dar clase con la boca cerrada. Universidad de Valencia.

Porlán, R. (2017). Enseñanza universitaria. Cómo mejorarla. Madrid: Ediciones Morata, S.L. 